\title{
Concordance to links with unknotted components
}

\author{
JAE CHOON CHA \\ DANIEL RUBERMAN
}

\begin{abstract}
We show that there are topologically slice links whose individual components are smoothly concordant to the unknot, but which are not smoothly concordant to any link with unknotted components. We also give generalizations in the topological category regarding components of prescribed Alexander polynomials. The main tools are covering link calculus, algebraic invariants of rational knot concordance theory, and the correction term of Heegaard Floer homology.
\end{abstract}

57M25; 57M27, 57N70

\section{Introduction}

This paper addresses the following question which arises naturally in the study of link concordance: to what extent can a component of a given link vary under link concordance? We have an obvious necessary condition-for a given link $L$, if a knot $K$ appears as a component of a link which is concordant to $L$, then $K$ is concordant to the corresponding component of $L$. Therefore a natural question is whether the converse holds: if $K$ is concordant to, say the first component of $L$, is there a link concordant to $L$ which has $K$ as the first component?

In general, there is no such link. In the case when $K$ is unknotted, Cochran [10] (using techniques introduced in [9]) and Cochran-Orr [11; 12] showed:

Theorem 1.1 There are links that have components smoothly concordant to the unknot but are not topologically concordant to any link with unknotted components.

This shows that the answer is negative in both topological and smooth category. In this paper, we investigate the difference between the smooth and topological cases, and generalize Theorem 1.1 to obstruct concordances involving different knot types. In the smooth case, we refine the result by giving examples which are topologically trivial:

Theorem 1.2 There are topologically slice links that have smoothly slice components but are not smoothly concordant to any link with unknotted components. 
In fact, there is no concordance to a link having components with trivial Alexander polynomial.

We also address the general case about concordance to links with an arbitrarily given component, in place of an unknotted component. In the topological setting, we considerably extend Theorem 1.1, giving obstructions that detect links not concordant to any link whose first component belongs to a given collection of Alexander polynomials:

Theorem 1.3 For any finite collection $D$ of classical Alexander polynomials of knots and for any knot $J_{0}$ with $\Delta_{J_{0}}(t) \in D$, there are links $L=K_{1} \cup K_{2}$ satisfying the following:

(1) The first component $K_{1}$ of $L$ is smoothly concordant to the given $J_{0}$.

(2) $L$ is not topologically concordant to any link $L^{\prime}=K_{1}^{\prime} \cup K_{2}^{\prime}$ with $\Delta_{K_{1}^{\prime}}(t) \in D$.

We give a smooth refinement of Theorem 1.3 by providing examples of links which are topologically concordant to a link with first component $J_{0}$. See Theorem 3.5 for details.

To prove the above results, we give general obstructions by combining several known techniques. In particular the main ingredients are the following: covering link calculus as used in Cochran-Orr [12] and formulated in Cha-Kim [4] (see also ChaLivingston [8], Van Cott [22] and Levine [19]), invariants of rational knot concordance (see Cha [3] and also Cochran-Orr [12] and Cha-Ko [6]), and the $d$-invariant (or correction term) of Heegaard Floer homology (see Ozsváth-Szabó [21], and also JabukaNaik [18], Grigsby-Ruberman-Strle [16] and Hedden-Livingston-Ruberman [17]).

The obstructions used to prove Theorems 1.2 and 1.3 are described in Section 2. See (respectively) Theorems 2.7 and 2.4.

In Section 3, we present explicit examples of links. The topological and smooth cases are dealt with in Theorem 3.1 and Theorem 3.5, respectively.

In Section 4, we investigate the nontriviality of rational homology cobordism groups modulo the classes of $\mathbb{Z}_{q}$-homology spheres using the 3-manifolds associated to our link examples.

\section{Links with components having given Alexander polynomi- als}

We consider ordered links, namely the components of a link are given a preferred labeling by integers $1, \ldots, m$. We also always assume that links are oriented. We say 
two $m$-component links $L=K_{1} \cup \ldots \cup K_{m}$ and $L^{\prime}=K_{1}^{\prime} \cup \ldots \cup K_{m}^{\prime}$ in $S^{3}$ are topologically (resp. smoothly) concordant if there are disjoint locally flat (resp. smooth) cylinders $C_{i}(i=1, \ldots, m)$ embedded in $S^{3} \times[0,1]$ with $\partial C_{i}=K_{i} \times 0 \cup-K_{i}^{\prime} \times 1$.

To simplify the notation we consider only the case of two-component links. We remark that all the arguments and results apply to the case of any number of components $(\geq 2)$ as well as the two-component case.

For a link $L=K_{1} \cup K_{2}$, we consider covering links as formulated and used in ChaKim [4] (see also Cochran-Orr [12], Cha-Livingston-Ruberman [8], Van Cott [22] and Levine [19]). In particular, we make use of the following special case. Suppose $d$ is a power of a prime $p$ and let $Y_{L}$ (or just $Y$ if the link $L$ is understood) be the $d$-fold cyclic branched cover of $S^{3}$ along $K_{1}$, the first component of the given link $L$. It is known that $Y_{L}$ is a $\mathbb{Z}_{p}$-homology sphere. (In this paper $\mathbb{Z}_{p}$ denotes $\mathbb{Z} / p \mathbb{Z}$.) If $L$ has linking number zero, the pre-image of $K_{2}$ in $Y$ consists of $p$ components. Choose a component $K_{L}$, which we call a covering knot of $L$. (For our purpose the choice of a component gives no ambiguity since the $\mathbb{Z}_{d}$-action permutes these component.)

Proposition 2.1 Suppose $L$ is topologically (resp. smoothly) concordant to a link $L^{\prime}=K_{1}^{\prime} \cup K_{2}^{\prime}$ with $\Delta_{K_{1}^{\prime}}(t)=1$. Then for any power $d$ of an arbitrary prime $p$, the $d$-fold covering knot $\left(Y_{L}, K_{L}\right)$ of $L$ described above is topologically (resp. smoothly) $\mathbb{Z}_{p}$-concordant to a knot in an integral homology sphere.

Here, as in Cha [3], two knots $(Y, J)$ and $\left(Y^{\prime}, J^{\prime}\right)$ in $R$-homology 3-spheres $Y$ and $Y^{\prime}$ are called topologically (resp. smoothly) $R$-concordant if there is a pair $(W, C)$ of a 4-manifold $W$ and an embedded cylinder $C$ satisfying $H_{*}(W, Y ; R)=0=$ $H_{*}\left(W, Y^{\prime} ; R\right)$ and $\partial(W, C)=(Y, J) \cup-\left(Y^{\prime}, J^{\prime}\right)$.

Proof It is known that if $L$ and $L^{\prime}$ are concordant, then the covering knot $K_{L}$ of $L$ is concordant to the corresponding covering knot $K_{L^{\prime}}$ of $L^{\prime}$ (for example, see the argument of Cha-Kim [4, Theorem 2.2]). The covering knot $K_{L^{\prime}}$ lies in the $d$-fold cyclic branched cover of $S^{3}$ along $K_{1}^{\prime}$, say $Y_{L^{\prime}}$, which is an integral homology sphere, since $K_{1}^{\prime}$ has Alexander polynomial one. This argument works in both smooth and topological cases.

A key fact used in the above proof of Proposition 2.1 is that the order of the homology of $Y_{L^{\prime}}$ is determined by the Alexander polynomial of the component $K_{1}^{\prime}$. Using this fact more extensively, Proposition 2.1 generalizes as follows.

For convenience of notation, for a polynomial $f(t)$ we denote

$$
\mathcal{R}_{d}(f)=\left|\prod_{k=0}^{d-1} f\left(e^{2 \pi k \sqrt{-1}}\right)\right| .
$$


It is well known that for a knot $K$ with Alexander polynomial $\Delta_{K}(t)$, the first homology of the $d$-fold cyclic branched cover of $\left(S^{3}, K\right)$ has order $\mathcal{R}_{d}\left(\Delta_{K}\right)$ (see Fox [13]). We note that $\mathcal{R}_{d}\left(\Delta_{K}\right)$ may be zero in general (in this case the first homology has nontrivial free part), but if $d$ is a prime power, $\mathcal{R}_{d}\left(\Delta_{K}\right)$ is always a positive integer.

Throughout this paper, we will write

$$
D=\left\{f_{1}(t), \ldots, f_{r}(t)\right\}
$$

for a finite collection of classical Alexander polynomials. By definition, these are integer coefficient polynomials satisfying $f_{i}(1)= \pm 1$ and $f\left(t^{-1}\right)=t^{2 g} f(t)$ for some $g \in \mathbb{N}$; we will make use of just the first condition. For a prime power $d=p^{a}$, define $\mathcal{P}_{d}(D)$ to be the set of primes that do not divide $\mathcal{R}_{d}\left(f_{i}\right)$ for all $i=1, \ldots, r$. One easily sees the following two properties:

(1) All but finitely many primes are in $\mathcal{P}_{d}(D)$.

(2) For $D=\{1\}, \mathcal{P}_{d}(D)$ is the set of all primes.

Theorem 2.2 Suppose a link $L$ is topologically (resp. smoothly) concordant to a link $L^{\prime}=K_{1}^{\prime} \cup K_{2}^{\prime}$ for which $\Delta_{K_{1}^{\prime}}(t)$ lies in $D$. Then for any prime power $d=p^{a}$, the $d$-fold covering knot $\left(Y_{L}, K_{L}\right)$ of $L$ described above is topologically (resp. smoothly) $\mathbb{Z}_{p}$-concordant to a knot in a 3-manifold which is a $\mathbb{Z}_{q}$-homology sphere for any $q \in \mathcal{P}_{d}(D)$.

Proof Observe that if $\Delta_{K_{1}^{\prime}}(t)=f_{i}(t) \in D$, then the $d$-fold covering link $K_{L^{\prime}}$ of $L^{\prime}$ lies in a 3-manifold $Y_{L^{\prime}}$ satisfying $\left|H_{1}\left(Y_{L^{\prime}}\right)\right|=\mathcal{R}_{d}\left(f_{i}\right)$, by the above discussion. By the hypothesis that $q$ does not divide $\mathcal{R}_{d}\left(f_{i}\right), Y_{L^{\prime}}$ is a $\mathbb{Z}_{q}$-homology sphere. The same argument as the proof of Proposition 2.1 concludes that $\left(Y_{L}, K_{L}\right)$ is $\mathbb{Z}_{p}$-concordant to $\left(Y_{L^{\prime}}, K_{L^{\prime}}\right)$.

\section{Obstructions from rational concordance theory: complexity of knots}

In Cochran-Orr [12], the notion of the complexity of a (codimension two) knot in a rational homology sphere was first introduced, and subsequently studied in Cha [3] and Cha-Ko [5] extensively. For the convenience of the reader, we recall the definition from [3, Definition 2.8]. For a knot $K$ in a rational homology 3-sphere $\Sigma$, an Alexander duality argument shows that $H_{1}(\Sigma-K ; \mathbb{Z}) /$ torsion $\cong \mathbb{Z}$. An essential difference from the integral homology sphere case is that the class of a meridian of $K$ does not necessarily generates $H_{1}(\Sigma-K ; \mathbb{Z}) /$ torsion though it is nonzero. The complexity of $K$ is defined to be the absolute value of the element in $\mathbb{Z} \cong H_{1}(\Sigma-K ; \mathbb{Z}) /$ torsion represented by the meridian. 
Obviously, a knot in an integral homology sphere has complexity one, by Alexander duality with integral coefficients. Therefore, from Proposition 2.1, we obtain the following:

Proposition 2.3 (Special case of Theorem 2.4) Suppose $L$ is topologically (resp. smoothly) concordant to a link $L^{\prime}=K_{1}^{\prime} \cup K_{2}^{\prime}$ with $\Delta_{K_{1}^{\prime}}(t)=1$. Then for any power $d$ of $p$, the $d$-fold covering knot $\left(Y_{L}, K_{L}\right)$ of $L$ described above is topologically (resp. smoothly) $\mathbb{Z}_{p}$-concordant to a knot with complexity one.

Using Theorem 2.2 in place of Proposition 2.1, we obtain the following generalization of Proposition 2.3:

Theorem 2.4 Suppose $L$ is topologically (resp. smoothly) concordant to a link $L^{\prime}=$ $K_{1}^{\prime} \cup K_{2}^{\prime}$ satisfying $\Delta_{K_{1}^{\prime}} \in D$. Then for any prime power $d=p^{r}$, the $d$-fold covering knot $\left(Y_{L}, K_{L}\right)$ of $L$ is topologically (resp. smoothly) $\mathbb{Z}_{p}$-concordant to a knot whose complexity is relatively prime to all $q \in \mathcal{P}_{d}(D)$.

Proof Suppose $q \in \mathcal{P}_{d}(D)$. By Theorem 2.2, $K_{L}$ is $\mathbb{Z}_{p}$-concordant to a knot in a $\mathbb{Z}_{q}$-homology sphere. The conclusion follows immediately from the following known fact, which is for example mentioned in [3, page 66]: the complexity of a knot $K$ in a $\mathbb{Z}_{q}$-homology sphere $\Sigma$ is relatively prime to $q$. A standard argument for this is to use the duality with $\mathbb{Z}_{q}$ coefficients-by Alexander duality $H_{1}\left(\Sigma-K ; \mathbb{Z}_{q}\right) \cong \mathbb{Z}_{q}$ is generated by a meridian of $K$, and thus the meridian represents an integer $\not \equiv 0(\bmod q)$ in $H_{1}(\Sigma-K) /$ torsion $\cong \mathbb{Z}$.

There are known obstructions to a knot in a rational homology sphere being topologically $\mathbb{Q}$-concordant to a knot with given complexity. Cochran and Orr first discovered an obstruction from the period of a signature function [12], which was reformulated in terms of Seifert matrices in Cha-Ko [5]. Also, the first author obtained further obstructions to being concordant to complexity one knots from torsion invariants of rational knot concordance, in his monograph [3, Theorem 4.17].

In the next section we will give examples to which one can apply the topological case of Theorem 2.4 (and Proposition 2.3) combined with these known obstructions to being concordant to a knot with given complexity.

\section{Obstructions from Heegaard Floer $\boldsymbol{d}$-invariants}

In case of smooth category, we obtain further obstructions from the $d$-invariants which are "correction terms" of Heegaard Floer homology of 3-manifolds (see OszváthSzabó [21]). For this purpose first we make the following observation on the $(1 / n)-$ surgery along a knot. First note that for a knot $K$ in a rational homology sphere, if $K$ 
has vanishing $(\mathbb{Q} / \mathbb{Z})$-valued self-linking, then there is a well-defined zero-framing on $K$ which is uniquely determined by the condition that $K$ and its parallel copy along the zero-framing has vanishing $\mathbb{Q}$-valued linking number. (The converse is also true. For more about this, see Cha [3] and Cha-Ko [5].) This enables us to identify a slope of surgery along $K$ with an element in $\mathbb{Q} \cup\{\infty\}$ when $K$ has vanishing $(\mathbb{Q} / \mathbb{Z})$-valued self-linking, exactly as in case of knots in $S^{3}$. In particular the $(a / b)$-surgery along $K$ is well-defined for $a / b \in \mathbb{Q}$.

We remark that if a component, say $K$, of a link in a $\mathbb{Z}_{p}$-homology sphere has vanishing $(\mathbb{Q} / \mathbb{Z})$-valued self-linking, then any component of a covering link of $L$ that projects to $K$ has vanishing $(\mathbb{Q} / \mathbb{Z})$-valued self-linking (see Cha-Kim [4, Section 2], and also Cha [3]). In particular, for a link $L$ in $S^{3}$, our covering knot $\left(Y_{L}, K_{L}\right)$ has vanishing $(\mathbb{Q} / \mathbb{Z})$-valued self-linking. It follows that the $(a / b)$-surgery along $K_{L}$ is well-defined.

Proposition 2.5 Let $R$ be a subring of $\mathbb{Q}$ or $\mathbb{Z}_{p}$. Suppose $K$ is a knot in an $R$ homology sphere with vanishing $(\mathbb{Q} / \mathbb{Z})$-valued self-linking. If $K$ is $R$-concordant to a knot in a $\mathbb{Z}_{q}$-homology sphere, then the $(a / b)$-surgery manifold of $K$ is $R$ homology cobordant to a $\mathbb{Z}_{q}$-homology sphere for any a relatively prime to $q$.

Proof We may assume $R$ is a subring of $\mathbb{Q}$, since we can replace $\mathbb{Z}_{p}$ with $\mathbb{Z}_{(p)}$, the ring of integers localized at $p$. The rational valued linking number is invariant under rational concordance (for example, see [5] and [3]). Therefore, if $(W, C)$ is an $R$-concordance between two knots, then (compare Gordon [15]) by removing a tubular neighborhood of $C$ from $W$ and filling in it with $S^{1} \times D^{2} \times[0,1]$ along the $(a / b)$-framing, we obtain an $R$-homology cobordism between their $(a / b)$-surgery manifolds. The proof is completed by observing that if a knot is in a $\mathbb{Z}_{q}$-homology sphere and $(a, q)=1$, then the $(a / b)$-surgery along the knot is again a $\mathbb{Z}_{q}$-homology sphere.

It was observed in Hedden-Livingston-Ruberman [17] that the Ozsváth-Szabó $d-$ invariant [21] gives an obstruction to being smoothly $\mathbb{Q}$-homology cobordant to an integral homology sphere. To simplify the discussion of $\operatorname{Spin}^{c}$ structures, we restrict to the case of $\mathbb{Z}_{2}$-homology spheres and homology cobordisms. For a $\mathbb{Z}_{2}$-homology 3-sphere $Y$, the composition

$$
\operatorname{Spin}^{c}(Y) \stackrel{c_{1}}{\longrightarrow} H^{2}(Y) \stackrel{\mathrm{PD}}{\longrightarrow} H_{1}(Y)
$$

of the first Chern class and Poincaré duality induces a bijection that takes the unique Spin structure on $Y$ to $0 \in H_{1}(Y)$. In the remainder of the paper, we use this bijection to label $\operatorname{Spin}^{c}$ structures by elements of $H_{1}(Y)$. 
For $s \in H_{1}(Y)$, denote by $d(Y, s)$ the correction term invariant defined from the $\mathbb{Q}$-valued grading of the Heegaard Floer homology of $Y$. Let

$$
\bar{d}(Y, s)=d(Y, s)-d(Y, 0) .
$$

Recall that a torsion abelian group $G$ decomposes into an inner direct sum $G=\bigoplus_{p} G_{p}$ of $p$-primary summands

$$
G_{p}=\left\{x \in G \mid p^{N} x=0 \text { for some } N \geq 0\right\}
$$

where $p$ runs over all primes. We remark that if $G$ is finitely generated, that $\left|G_{p}\right|$ is equal to the maximal power of $p$ dividing the order of $G$. Also, if $A$ is a subgroup of $G_{p}$, then obviously $A_{p}=A \cap G_{p}$.

For our purpose the following generalization of Hedden-Livingston-Ruberman [17, Theorem 3.2] is useful. For similar applications of $d$ and $\bar{d}$, see, for example, JabukaNaik [18] and Grigsby-Ruberman-Strle [16].

Proposition 2.6 Suppose $q$ is a prime and $Y$ is a $\mathbb{Z}_{2}$-homology 3-sphere which is $\mathbb{Z}_{2}$-homology cobordant to a $\mathbb{Z}_{q}$-homology sphere. Then there is a subgroup $H$ of the $q$-primary part $H_{1}(Y)_{q}$ satisfying $|H|^{2}=\left|H_{1}(Y)_{q}\right|$ and $\bar{d}(Y, s)=0$ for any $s \in H$.

We note that Proposition 2.6 easily applies to a 3-manifold $Y$ which is a boundary component of a $\mathbb{Z}_{2}$-homology punctured 4-ball $W$ with $H_{1}\left(\partial W-Y ; \mathbb{Z}_{q}\right)=0$, by tunneling $W$ to join components of $\partial W-Y$.

Proof Suppose $Y^{\prime}$ is a $\mathbb{Z}_{q}$-homology sphere and $Y$ is $\mathbb{Z}_{2}$-homology cobordant to $Y^{\prime}$. Denote $G=H_{1}(Y) \oplus H_{1}\left(Y^{\prime}\right)=H_{1}(\partial W)$. Let $A$ be the image of the boundary map $H_{2}(W, \partial W) \rightarrow G$ which is identified with $\operatorname{Spin}^{c}(W)=H^{2}(W) \rightarrow H^{2}(\partial W)=$ $\operatorname{Spin}^{c}(\partial W)$ under Poincare duality. Namely, $A$ consists of the $\operatorname{Spin}^{c}$-structures of $\partial W$ that extends to $W$. By Ozsváth-Szabó [21, Theorem 1.2], $d\left(\partial W,\left(s, s^{\prime}\right)\right)=$ $d(Y, s)-d\left(Y^{\prime}, s^{\prime}\right)=0$ whenever $\left(s, s^{\prime}\right) \in A \subset G$. Also, by the argument of CassonGordon [2], $|A|^{2}=|G|$.

Consider the $q$-primary parts $G_{q}$ and $A_{q}$. Since $\left|H_{1}\left(Y^{\prime}\right)\right|$ is relatively prime to $q$, we have $G_{q}=H_{1}(Y)_{q}$ and $A_{q} \subset G_{q} \subset H_{1}(Y)$. Also, $|A|^{2}=|G|$ implies $\left|A_{q}\right|^{2}=\left|H_{1}(Y)_{q}\right|$, by using the remark before Proposition 2.6.

Now, for $s \in A_{q} \subset H_{1}(Y)$, since $(s, 0) \subset A \subset G$, we have $d(Y, s)-d\left(Y^{\prime}, 0\right)=0$. In particular $d(Y, 0)-d\left(Y^{\prime}, 0\right)=0$. It follows that $\bar{d}(Y, s)=d(Y, s)-d(Y, 0)=0$ for any $s \in H_{1}(Y)_{q}$. 
As remarked above, if $d$ is a power of 2 , then the $d$-fold branched cover of $S^{3}$ along a knot is a $\mathbb{Z}_{2}$-homology sphere. Hence we may combine Theorem 2.2 and Propositions 2.5 and 2.6, to obtain the following:

Theorem 2.7 Suppose $L$ is smoothly concordant to a link $L^{\prime}=K_{1}^{\prime} \cup K_{2}^{\prime}$ satisfying $\Delta_{K_{1}^{\prime}}(t) \in D$. Suppose $Y$ is the $(a / b)$-surgery manifold of the $d$-fold covering knot $\left(Y_{L}, K_{L}\right)$ of $L$. If $d$ is a power of two, $q \in \mathcal{P}_{d}(D)$, and $a$ is relatively prime to $q$, then there is a subgroup $H$ of the $q$-primary part $\subset H_{1}(Y)_{q}$ such that $|H|^{2}=\left|H_{1}(Y)_{q}\right|$ and $\bar{d}(Y, s)=0$ for any $s \in H$.

We remark that if $D=\{1\}$, namely if $L$ is assumed to be concordant to $L^{\prime}$ with $\Delta_{K_{1}^{\prime}}(t)=1$ in Theorem 2.7, then the condition " $q \in \mathcal{P}_{d}(D)$ " is replaced with " $q$ is any prime" in the conclusion.

We also remark that our subgroup $H$ in Theorem 2.7 has the property that the $(\mathbb{Q} / \mathbb{Z})$ valued linking form of $Y$ vanishes on $H \times H$. In this paper we do not use this.

\section{Examples}

Our main examples are of the following form. Fix a knot $J_{0}$. For an integer $m$ and a knot $J$, let $L(m, J)$ be the link illustrated in Figure 1. Here $J$ is a knot that will be specified later, and $-m$ denotes negative $m$ full twists between the obvious two vertical bands, in such a way that no self-twisting is added on each band. We remark that in the case of unknotted $J_{0}$, the link $L(m, J)$ was first considered in Cochran-Orr [12] to give examples which are not concordant to boundary links. In [12] it is shown that $L(m, J)$ is a homology boundary link for unknotted $J_{0}$. The same method shows that $L(m, J)$ is a homology boundary link for any $J_{0}$. Consequently $L(m, J)$ has vanishing $\bar{\mu}$-invariants.

Figure 2 illustrates a surgery diagram of the 2-fold covering knot $\left(Y_{L(m, J)}, K_{L(m, J)}\right)$, which is obtained from Figure 1 by applying the technique of Akbulut and Kirby [1]. The ambient space $Y_{L(m, J)}$ is the connected sum of the double branched cover of $J_{0}$ and the double branched cover of $K_{1}$. The latter is the result of surgery on $S^{3}$ along the two 0-framed circles in Figure 2.

Note that $K_{1}$ and $J_{0}$ are smoothly concordant, as knots. One way to see this is as follows: if one performs a band surgery on $K_{1}$ along the dotted $\operatorname{arc} \beta$ in Figure 1 (and forgets $K_{2}$ ), then $K_{1}$ becomes a split union of $J_{0}$ and an unknotted circle. 


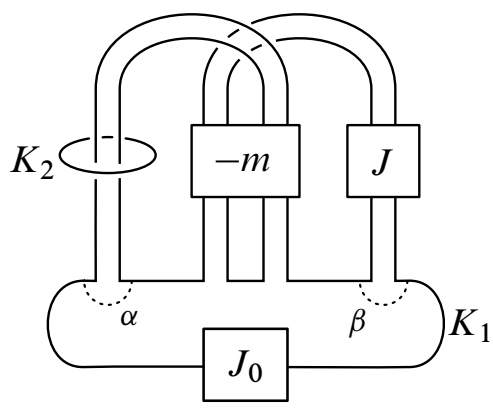

Figure 1. The link $L(m, J)$.

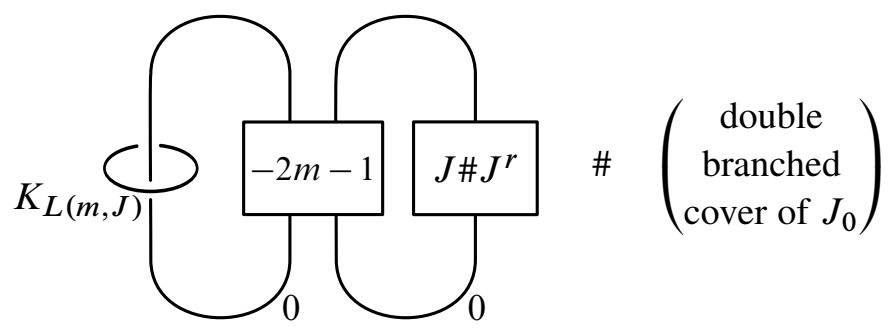

Figure 2. The covering knot $K_{L(m, J)}$ in $Y_{L(m, J)}$

\subsection{Topological examples}

In this subsection we prove the following result:

Theorem 3.1 For any finite collection $D$ of classical Alexander polynomials of knots and for any knot $J_{0}$ with $\Delta_{J_{0}}(t) \in D$, there are links $L=K_{1} \cup K_{2}$ satisfying the following:

(1) The first component $K_{1}$ of $L$ is smoothly concordant to the given $J_{0}$.

(2) $L$ is not topologically concordant to any link $L^{\prime}=K_{1}^{\prime} \cup K_{2}^{\prime}$ with $\Delta_{K_{1}^{\prime}}(t) \in D$.

Note that our example $L(m, J)$ in Figure 1 satisfies Theorem $3.1(1)$ as observed above. In what follows we will show that for an appropriate choice of $J$ that will be given later $L(m, J)$ satisfies Theorem $3.1(2)$.

We recall that for a knot $K$ in a rational homology sphere $Y$ with vanishing $(\mathbb{Q} / \mathbb{Z})$ valued self-linking, there is a signature invariant of $K$ (see Cochran-Orr [12], ChaKo [5] and Cha [3]). For readers who are not familiar with this, we give a description 
in terms of Seifert matrices, which was first given in Cha-Ko [5]. (The description below differs from that in [5] by a reparametrization by a factor of $2 \pi$.)

It is known that if $K$ has vanishing $(\mathbb{Q} / \mathbb{Z})$-valued linking, then there is a generalized Seifert surface for $K$, which is defined to be a properly embedded surface $F$ in the exterior of $K$ whose boundary consists of $c$ parallel copies of $K$ taken along the 0 -framing. It is known that there exists such $F$ if and only if $c$ is a multiple of the complexity of $K$ (see [5] and [3, Chapter 2]). A Seifert matrix $A$ for the generalized Seifert surface $F$ is defined as usual, using the $\mathbb{Q}$-valued linking number in $Y$ in place of the ordinary linking number. Then for $\theta \in \mathbb{R}, \delta_{K}(\theta)$ is defined to be the jump at $t=\theta / c$ of the signature function

$$
\sigma_{A}(t)=\operatorname{sign}\left(\left(1-e^{2 \pi t \sqrt{-1}}\right) A+\left(1-e^{-2 \pi t \sqrt{-1}}\right) A^{T}\right) .
$$

We regard $\delta_{K}$ as a function $\mathbb{R} \rightarrow \mathbb{Z}$. It is known that $\delta_{K}$ is an invariant under rational concordance $[12 ; 5 ; 3]$. We note that the reparametrization by the factor of $1 / c$ given in the definition is essential in proving the invariance.

For our purpose the following property is useful. We say that $\delta_{K}$ has period $c$ if $\delta_{K}(\theta)=\delta_{K}(\theta+c)$ for all $\theta$.

Theorem 3.2 (Cochran-Orr [12], Cha-Ko [5], Cha [3]) If a knot $K$ in a rational homology sphere is rationally concordant to a knot with complexity $c$, then $\delta_{K}(\theta)$ has period $c$.

We remark that since the set $\left\{\theta \in \mathbb{R} \mid \delta_{K}(\theta) \neq 0\right\}$ of non-vanishing points is discrete, there is a minimal period, say $c_{0}>0$, of $\delta_{K}(\theta)$ such that any period of $\delta_{K}(\theta)$ is an integer multiple of $c_{0}$. (For, otherwise the set of periods of $\delta_{K}$ is dense in $\mathbb{R}$.)

Proof of Theorem 3.1 As observed in the beginning of this section, the first component $K_{1}$ of our $L(m, J)$ is concordant to $J_{0}$, regardless of the choice of $J$. This shows (1).

Choose a knot $J$ whose signature jump function $\delta_{J}(\theta)$ has minimal period one. For example, one can take the trefoil knot $3_{1}$ as $J$, since $\delta_{3_{1}}(\theta)= \pm 2$ exactly for $\theta \equiv \pm 1 / 6(\bmod 1)$. We note that many more such knots exist, for example, by appealing to Cha-Livingston [7].

Choose an odd prime $q \in \mathcal{P}_{2}(D)$ and let $m=(q-1) / 2$. Consider the link $L=$ $L(m, J)$ and the 2 -fold covering knot $\left(Y_{L}, K_{L}\right)$ of $L$. The realization argument of Cha [3, Section 4.1.2] (see also Cha-Ko [5, Example, page 1179]) shows that $\delta_{K_{L}}(\theta)=\delta_{J_{\# J^{r}}}(\theta / q)$. Since $\delta_{J_{\#} J^{r}}(\theta)=\delta_{J}(\theta)+\delta_{J^{r}}(\theta)=2 \delta_{J}(\theta)$, it follows that $\delta_{K_{L}}(\theta)$ has minimal period $q$. 
Therefore, no period of $\delta_{K_{L}}(\theta)$ is relatively prime to $q$. By Theorem 3.2, $K_{L}$ is not rationally concordant to any knot whose complexity is relatively prime to $q$. By Theorem 2.4 it follows that $L$ is not concordant to any link $L^{\prime}=K_{1}^{\prime} \cup K_{2}^{\prime}$ for which $\Delta_{K_{1}^{\prime}}(t) \in D$.

Theorem 1.1 stated in the introduction is an immediate consequence of the special case of Theorem 3.1 for $D=\{1\}$ and $J_{0}=$ unknot.

Remark 3.3 Another class of links that may be shown to satisfy Theorem 1.1 by our method is given in Cha-Ko [6, Figure 2]. In the proof of [6, Theorem 3.3], it was shown that these links have covering links which are not concordant to a complexity one knot. Therefore, our method shows that these satisfy Theorem 1.1. An interesting property of the links given in [6, Figure 2] is that these are mutants of ribbon links. Therefore these links are not distinguished from smoothly slice links by any invariants preserved under mutation.

Remark 3.4 After we had announced our main results, Charles Livingston informed us that he found an alternative approach using Casson-Gordon invariants as in Livingston [20] to show Theorem 1.1 for the same examples.

\subsection{Topologically slice smooth examples}

Theorem 3.5 For any finite collection $D$ of classical Alexander polynomials of knots and for any knot $J_{0}$ with $\Delta_{J_{0}}(t) \in D$, there are links $L=K_{1} \cup K_{2}$ satisfying the following:

(1) L is topologically concordant to the split union of $J_{0}$ and an unknotted circle.

(2) The first component $K_{1}$ of $L$ is smoothly concordant to the given $J_{0}$.

(3) $L$ is not smoothly concordant to any link $L^{\prime}=K_{1}^{\prime} \cup K_{2}^{\prime}$ with $\Delta_{K_{1}^{\prime}}(t) \in D$.

Proof Again we consider $L=L(m, J)$, now with the extra condition that $J$ be topologically slice. As before, $L$ satisfies (2). Also note that $L$ satisfies (1), since one obtains the split union $J_{0} \cup$ (unknot) $\cup K_{2}$ from $L$ ( $m$, unknot) by a band surgery on $K_{1}$ along the dotted arc $\alpha$ in Figure 1.

Let $Y=Y(m, J)$ be the 3-manifold obtained by performing 1-surgery on $Y_{L}$ along $K_{L}$. Recall that we need to be careful with the framing: the surgery coefficient is determined with respect to the zero-framing on $K_{L}$ in $Y_{L}$, which is defined in terms of the $\mathbb{Q}$-valued linking number in $Y_{L}$, as in the previous section. In our case the zero-framing on $K_{L}$ in $Y_{L}$ is identical with the usual zero-framing on $K_{L}$ in the 
surgery description of $Y_{L}$ given in Figure 2. This can be verified, for example, by computing the $\mathbb{Q}$-valued linking number of $K_{L}$ and its preferred longitude using a formula given in Cha-Ko [5, Theorem 3.1]. Alternatively, a surface in $Y_{L}$ which is bounded by $(2 m+1)$ parallel copies of $K_{L}$ is obtained from a capped-off Seifert surface for $J \# J^{r}$ by attaching $(2 m+1)$ tubes, and then the framing induced by this surface, which is the zero-framing on $K_{L}$ in $Y_{L}$, is seen to be equal to the ordinary zero-framing.

Therefore Figure 2 with surgery coefficient 1 on $K_{L}$ becomes a surgery diagram for the 1-surgery manifold $Y$. Let $M_{0}$ be the double branched cover of $J_{0}$, and let $M$ be the $(2 m+1)^{2}$-surgery manifold of the knot $T(2 m+1,2 m) \# J \# J^{r}$. Here $T(a, b)$ denotes the $(a, b)$-torus knot. As illustrated in Figure 3, one sees that $Y$ is diffeomorphic to $M_{0} \# M$.
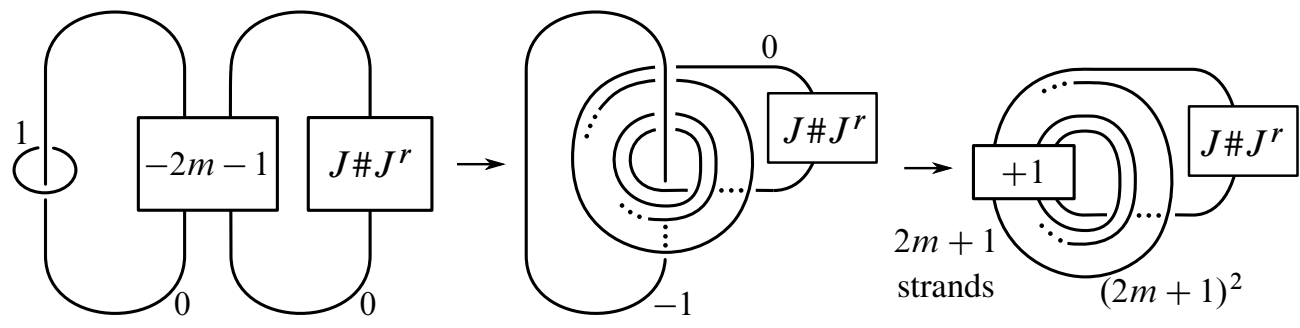

Figure 3. 1-surgery along $K_{L}$.

Now we specify $m$ and $J$. For the given collection $D$, choose an odd prime $q \in \mathcal{P}_{2}(D)$, and let $m=(q-1) / 2$. Let $J$ be the connected sum of $(3 m+1) / 2$ copies of the positive Whitehead double of the right-hand trefoil knot. The knot $J$ is topologically slice by Freedman's theorem [14].

It is easily seen that $H_{1}(Y)=H_{1}\left(M_{0}\right) \oplus H_{1}(M)$ and $H_{1}(M)=\left(\mathbb{Z}_{2 m+1}\right)^{2}=\left(\mathbb{Z}_{q}\right)^{2}$. Since $q \in \mathcal{P}_{2}(D)$ and $\Delta_{J_{0}}(t) \in D,\left|H_{1}\left(M_{0}\right)\right|$ is relatively prime to $q$ by our definition of $\mathcal{P}_{2}(D)$ (see the discussion in Section 2). Therefore $H_{1}(Y)_{q}=H_{1}(M)$, and the subgroup $H=q \mathbb{Z}_{q^{2}}$ generated by $q \in H_{1}(M)=\mathbb{Z}_{q^{2}}$ is the unique subgroup satisfying $|H|^{2}=\left|H_{1}(Y)_{q}\right|$. By Theorem 2.7, for any $s \in H \subset H_{1}(Y)$, we have $\bar{d}(Y, s)=0$ if $L$ is concordant to a link $L^{\prime}=K_{1}^{\prime} \cup K_{2}^{\prime}$ satisfying $\Delta_{K_{1}^{\prime}}(t) \in D$. Note that $\bar{d}(Y, s)=\bar{d}(M, s)$ since $s$ lies in $H_{1}(M) \subset H_{1}\left(M_{0}\right) \oplus H_{1}(M)=H_{1}(Y)$.

In [17, Theorem 6.4], Hedden, Livingston and the second author proved that for the above $J, \bar{d}(M, q) \geq 2$. Since $q \in H$, the conclusion follows from this.

Theorem 1.2 in the introduction is an immediate consequence of the special case of Theorem 3.5 for $D=\{1\}$ and $J_{0}=$ unknot. 


\section{Rational homology cobordism groups and $\mathbb{Z}_{p}$-homology spheres}

In [17], a calculation of $d$-invariants is used to elucidate the structure of smooth rational homology cobordism group modulo integral homology spheres. In this section, we investigate the more general case of $\mathbb{Z}_{p}$-homology spheres instead of integral homology spheres. For this purpose we use Proposition 2.6, which gives obstructions to being rational homology cobordant to $\mathbb{Z}_{p}$-homology spheres.

For simplicity we first consider the case of $\mathbb{Z}_{2}$-homology spheres. Let $\Omega$ be the group of smooth rational homology cobordism classes of $\mathbb{Z}_{2}$-homology spheres. Let $\Omega_{T}$ be the kernel of the natural homomorphism of $\Omega$ into its topological analogue, namely, $\Omega_{T}$ is the subgroup in $\Omega$ that consists of all $\mathbb{Z}_{2}$-homology spheres which are topologically rational homology cobordant to $S^{3}$. For a commutative ring $R$, let $\Omega_{T}^{R}$ be the subgroup of $\Omega_{T}$ generated by (the classes of) $R$-homology spheres in $\Omega_{T}$.

Theorem 4.1 For any odd prime $q, \Omega_{T} / \Omega_{T}^{\mathbb{Z}_{q}}$ is nontrivial.

Proof In the proof of Theorem 3.5, we observed that the $\mathbb{Z}_{2}$-homology sphere $M$ has the following properties: (i) The subgroup $H$ generated by $q \in H_{1}(M)=H_{1}(M)_{q}$ is the unique one satisfying $|H|^{2}=\left|H_{1}(M)_{q}\right|$, and (ii) $\bar{d}(M, q) \geq 2$. By Proposition 2.6, it follows that $M$ is not rational homology cobordant to any $\mathbb{Z}_{q}$-homology sphere.

Remark 4.2 We can also think of, in place of $\Omega$, the smooth spin rational homology cobordism group of spin rational homology 3-spheres, and spin analogues of the subgroups $\Omega_{T}$ and $\Omega_{T}^{\mathbb{Z}_{q}}$. Our argument above shows that Theorem 4.1 also holds for this case as well, since one can canonically identify $\operatorname{Spin}^{c}(Y)$ with $H^{2}(Y)$ for manifolds $Y$ with a chosen spin structure.

As a corollary we obtain that $\Omega_{T} / \Omega_{T}^{\mathbb{Z}}$ is nontrivial, which is a consequence of [17, Theorem 7.1]. In fact [17, Theorem 7.1] says that $\Omega_{T} / \Omega_{T}^{\mathbb{Z}}$ has infinite rank. Regarding this and our theorem above, one may ask the following question: for an odd prime $q$, does $\Omega_{T} / \Omega_{T}^{\mathbb{Z}_{q}}$ have infinite rank?

Acknowledgments We thank Tim Cochran and Kent Orr for informative comments on the first version of this paper, and for providing references to earlier work in the area. We also thank Charles Livingston for his comments. The first author was supported by the National Research Foundation of Korea (NRF) grant funded by the Korea government (MEST), No. 2010-0011629 and 2010-0029638. The second author was partially supported by NSF Grants DMS-0804760 and DMS-1105234, and by JSPS Grant-in-Aid No. 19340015. 


\section{References}

[1] S Akbulut, R Kirby, Branched covers of surfaces in 4-manifolds, Math. Ann. 252 (1979/80) 111-131 MR593626

[2] A J Casson, CM Gordon, Cobordism of classical knots, from: "À la recherche de la topologie perdue”, Progr. Math. 62, Birkhäuser, Boston, MA (1986) 181-199 MR900252 With an appendix by P. M. Gilmer

[3] J C Cha, The structure of the rational concordance group of knots, Mem. Amer. Math. Soc. 189 (2007) x+95 MR2343079

[4] J C Cha, T Kim, Covering link calculus and iterated Bing doubles, Geom. Topol. 12 (2008) 2173-2201 MR2431018

[5] J C Cha, K H Ko, Signatures of links in rational homology spheres, Topology 41 (2002) 1161-1182 MR1923217

[6] J C Cha, K H Ko, Signature invariants of covering links, Trans. Amer. Math. Soc. 358 (2006) 3399-3412 MR2218981

[7] J C Cha, C Livingston, Knot signature functions are independent, Proc. Amer. Math. Soc. 132 (2004) 2809-2816 MR2054808

[8] J C Cha, C Livingston, D Ruberman, Algebraic and Heegaard-Floer invariants of knots with slice Bing doubles, Math. Proc. Cambridge Philos. Soc. 144 (2008) 403-410 MR2405897

[9] T D Cochran, Geometric invariants of link cobordism, Comment. Math. Helv. 60 (1985) 291-311 MR800009

[10] T D Cochran, $k$-cobordism for links in $S^{3}$, Trans. Amer. Math. Soc. 327 (1991) 641-654 MR1055569

[11] T D Cochran, K E Orr, Not all links are concordant to boundary links, Bull. Amer. Math. Soc. 23 (1990) 99-106 MR1031581

[12] T D Cochran, K E Orr, Not all links are concordant to boundary links, Ann. of Math. 138 (1993) 519-554 MR1247992

[13] R H Fox, Free differential calculus III: Subgroups, Ann. of Math. 64 (1956) 407-419 MR0095876

[14] M H Freedman, The disk theorem for four-dimensional manifolds, from: "Proceedings of the International Congress of Mathematicians, Vol. 1, 2 (Warsaw, 1983)", PWN, Warsaw (1984) 647-663 MR804721

[15] C M Gordon, Knots, homology spheres, and contractible 4-manifolds, Topology 14 (1975) 151-172 MR0402762

[16] J E Grigsby, D Ruberman, S Strle, Knot concordance and Heegaard Floer homology invariants in branched covers, Geom. Topol. 12 (2008) 2249-2275 MR2443966 
[17] M Hedden, C Livingston, D Ruberman, Topologically slice knots with nontrivial Alexander polynomial, to appear in Adv. Math. arXiv:1001.1538

[18] S Jabuka, S Naik, Order in the concordance group and Heegaard Floer homology, Geom. Topol. 11 (2007) 979-994 MR2326940

[19] A S Levine, Slicing mixed Bing-Whitehead doubles, to appear in J. Topology arXiv: 0912.5222

[20] C Livingston, Links not concordant to boundary links, Proc. Amer. Math. Soc. 110 (1990) 1129-1131 MR1031670

[21] P Ozsváth, Z Szabó, Absolutely graded Floer homologies and intersection forms for four-manifolds with boundary, Adv. Math. 173 (2003) 179-261 MR1957829

[22] C Van Cott, An obstruction to slicing iterated Bing doubles arXiv:0907.4948

Department of Mathematics and PMI, POSTECH

Pohang 790-784, Republic of Korea

Department of Mathematics, Brandeis University

Waltham, MA 02454-9110, USA

jccha@postech.ac.kr, ruberman@brandeis.edu

http://www.postech.ac.kr/ jccha/,

http://people.brandeis.edu/ ruberman/

Received: 13 April 2011 Revised: 27 January 2012 